\title{
A influência da aprendizagem autorregulada na mestria escolar
}

\section{The influence of self-regulated learning in academic achievement}

\section{Maria Olímpia Almeida de Paiva*}

CIPE - Centro de Investigação em Psicologia e Educação. Escola Secundária Alexandre Herculano - Porto, Portugal

\author{
Abílio Afonso Lourenço** \\ CIPE - Centro de Investigação em Psicologia e Educação. Escola Secundária \\ Alexandre Herculano - Porto, Portugal
}

\begin{abstract}
RESUMO
Na última década, as investigações efetuadas no âmbito da autorregulação da aprendizagem revelaram um grande impacto na melhoria do rendimento escolar dos alunos.

Esta investigação teve como objetivo estudar a influência da disrupção percebida na autorregulação da aprendizagem e a influência desta no rendimento escolar dos alunos, numa escola pública do norte de Portugal. Para avaliar os construtos utilizaram-se dois instrumentos - o Inventário de Processos de Autorregulação da Aprendizagem e o Questionário dos Comportamentos Disruptivos Percebidos. Foram analisadas as respostas de 147 alunos ( 77 do sexo masculino e 70 do feminino), do 3. Ciclo do Ensino Básico, com idades compreendidas entre os 12 e 19 anos.

Constatou-se que os alunos que apresentam menor percepção dos comportamentos disruptivos são mais autorregulados na sua aprendizagem e obtêm melhores notas (Língua Portuguesa e Matemática), o que acentua a importância destes construtos no sucesso escolar. São discutidas implicações educativas nestes níveis de ensino.
\end{abstract}

Palavras-chave: Autorregulação da aprendizagem; rendimento acadêmico; disrupção percebida; ensino básico.

\footnotetext{
ABSTRACT

In the last decade investigations on self-regulated learning have proved to be fundamental to understanding the improvement of student achievement. The current investigation aimed to study the impact of perceived disruption in self-regulated learning, as well as its influence on the academic achievement of students in a state school in north of Portugal. To assess the constructs under study we used two questionnaires - the Inventory of Pupils' Self-regulatory Processes and Perceived Disruptive Behavior Questionnaire. The answers of 147 students, aged between 12-19 years old, were analyzed. This sample consisted of $77(52.4 \%)$ males and $70(47.6 \%)$ females of Middle School.
} 
It was found that students who have a lower perception of disruptive behavior are more self-regulating in their learning and get better grades (Mother Language and Math), which emphasizes the importance of these constructs on academic success. Educational implications are discussed at these levels of education.

Keywords: Self-regulated learning; academic achievement; perceived disruption; middle school.

\section{I ntrodução}

O rendimento académico é uma preocupação crescente de toda a comunidade educativa. No centro da problemática encontra-se o fracasso escolar e o correspondente abandono de muitos estudantes (ROSÁRIO; GONZÁLEZ-PIENDA; CEREZO; PINTO; FERREIRA; LOURENÇO; PAIVA, 2010). Ao observarmos a investigação produzida, nas últimas três décadas, sob o tópico da aprendizagem autorregulada, podemos concluir que este é um novo e importante construto explicativo dos processos de aprendizagem, com implicações evidentes no sucesso escolar (BANDURA, 2002; ROSÁRIO, 2004; ROSÁRIO; NÚÑEZ; GONZÁLEZ-PIENDA， 2004; SCHUNK; ERTMER, 2000; WINNE; NESBIT, 2010; ZIMMERMAN, 2000).

Sobre o construto da autorregulação da aprendizagem não existe uma definição simples e direta. Boekaerts e Corno (2005) sugeremnos que o processo de autorregulação do aluno pressupõe o domínio e gestão de fatores que se manifestam como elementos fundamentais para uma aprendizagem de qualidade e, consequentemente, da mestria escolar. É de salientar a importância do estabelecimento de objetivos nos diferentes momentos de aprendizagem, o envolvimento na tarefa, a planificação e a gestão adequada do tempo, o uso de estratégias apropriadas, a criação de um clima produtivo de trabalho, a utilização e aproveitamento eficaz dos recursos disponíveis, a monitorização das realizações, a antevisão dos resultados escolares e a procura de ajuda. Associado a estes fatores, é indispensável que o aluno tenha interiorizado crenças positivas sobre as suas capacidades e a qualidade da sua aprendizagem, como também sinta satisfação com o empenho pessoal desenvolvido (MCCOMBS, 1989).

Para Rosário (2004, p.37) a autorregulação da aprendizagem pode ser definida como: "um processo activo no qual os sujeitos estabelecem os objetivos que norteiam a sua aprendizagem tentando monitorizar, regular e controlar as suas cognições, motivação e comportamentos com o intuito de os alcançar". Deste modo, o mesmo autor refere, ainda, que a aprendizagem autorregulada implica um modelo dinâmico de aquisição do conhecimento, podendo ser considerada como um processo ativo, construtivo e orientado 
para objetivos sob a tutela da interacção dos recursos cognitivos, motivacionais e emocionais do aluno. Por seu lado, Zimmerman (2000) alude que este construto traduz a capacidade de os alunos desenvolverem conhecimento, competências estratégicas e comportamentos essenciais para incrementar as futuras aprendizagens, quer no contexto escolar, quer nas suas vivências cotidianas. Assim, em contexto de aprendizagem, a autorregulação relaciona-se com o processo que abrange a ativação e manutenção das cognições, atitudes e afetos dos alunos, planeados e ciclicamente ajustados com o objetivo de atingir as suas metas escolares (SCHUNK, 1994; ZIMMERMAN, 2000).

Relativamente ao comportamento escolar disruptivo pode ser entendido como "aquele que prejudica a aprendizagem dos alunos ou a eficácia do ambiente de ensino" (MERRETT; WHELDALL, 1984, p.87) ou, "aquele que vai contra as regras escolares, prejudicando as condições de aprendizagem, o ambiente de ensino ou 0 relacionamento das pessoas na escola" (VEIGA, 2001, p. 15).

Além das carências nos domínios sociais e emocionais, muitas crianças e adolescentes disruptivos também revelam dificuldades significativas na realização académica (EPSTEIN; KINDER; BURSUCK, 1989).

Os estudos de Epstein e colaboradores (1989) sobre o status acadêmico de adolescentes com problemas disruptivos, concluíram que, embora manifestem problemas de leitura, o seu insucesso acadêmico deve-se principalmente ao desvio das regras comportamentais em sala de aula. A própria aprendizagem é um instrumento vital na prevenção da disrupção e, como é recorrentemente referido pela literatura da área, a autorregulação da aprendizagem dos alunos é uma condição imprescindível para $O$ seu sucesso escolar (ZIMMERMAN, 2000; ZIMMERMAN; MARTINEZ-PONS, 1990). Como afirma Schunk (1994), os alunos autorregulados são intrinsicamente motivados para a aprendizagem e para o comportamento apropriado na sala de aula. Pelo contrário, os indivíduos com dificuldade na autorregulação da sua aprendizagem apresentam rendimentos escolares mais fracos (ZIMMERMAN; MARTINEZ-PONS; 1986; 1988) e revelam mais comportamentos problemáticos perante os seus professores (BRODY; STONEMAN; FLOR, 1996).

Para confirmar o objetivo da nossa investigação, relacionado com o modelo explicativo dos comportamentos disruptivos percebidos na compreensão da autorregulação da aprendizagem e a influência desta no rendimento dos alunos, usamos a análise de equações estruturais para obter evidência das relações causais entre as variáveis especificadas (BISQUERRA, 1989; NÚÑEZ, 1992). Assim, deste objetivo resulta a seguinte hipótese: 
- O modelo proposto representa as relações entre as variáveis existentes na nossa matriz empírica.

No que diz respeito às relações constantes no modelo hipotetizado assumimos, por parcimônia, que cada um dos construtos influencia apenas os seus itens específicos (BYRNE, 2001) e as relações entre variáveis exógenas e endógenas são representadas por um alfa (a) e entre as endógenas por um beta $(\beta)$. Desta forma, com base na revisão de literatura, foram estabelecidas as sub-hipóteses que orientam as especificações apresentadas:

H1 - O sexo feminino revela uma melhor percepção dos comportamentos disruptivos relativamente ao sexo oposto (CAPELA, 2003; PAIVA; LOURENÇO, 2009);

H2 - Os alunos do 9.. ano de escolaridade revelam menor percepção dos comportamentos disruptivos (CAPELA, 2003);

H3 - Os alunos com maior número de reprovações têm uma melhor percepção dos comportamentos disruptivos (PAIVA; LOURENÇO, 2009);

H4 - Um maior investimento no tempo de estudo, por parte dos alunos, influencia negativamente a percepção dos comportamentos disruptivos dos mesmos (PAIVA; LOURENÇO, 2009);

H5 - Os alunos que revelam metas escolares mais ambiciosas percepcionam melhor os comportamentos disruptivos (CAPELA, 2003; PAIVA; LOURENÇO, 2009);

H6 - Os alunos que apresentam uma melhor percepção sobre os comportamentos disruptivos são menos autorregulados na sua aprendizagem (CAPELA, 2003; ROSÁRIO; SOARES; MOURÃO; NÚÑEZ; GONZÁLEZ-PIENDA; SIMÕES; PINA, 2005);

$\mathrm{H} 7$ - Os alunos mais autorregulados na sua aprendizagem apresentam melhores notas escolares (LOURENÇO, 2008; ROSÁRIO; LOURENÇO; PAIVA; NÚÑEZ; GONZÁLEZ-PIENDA; VALLE, 2010);

HIM (hipótese resultante dos índices de modificação do modelo de equações estruturais) - Os alunos que investem mais horas no estudo apresentam uma maior frequência de comportamentos autorregulatórios na sua aprendizagem (ROSÁRIO et al, 2010).

\section{Método}

\subsection{Participantes}

Foi utilizada uma amostra de 147 alunos do ensino obrigatório português (7. , 8. e 9.. anos de escolaridade), distribuídos por nove turmas. Esta amostra, que corresponde ao universo dos alunos, foi recolhida numa escola secundária pública com 3. ciclo, do Porto Portugal, de perfil tipicamente urbano. 
Da amostra, 77 (52.4\%) alunos são rapazes e $70(47.6 \%)$ são raparigas. Frequentam o 7.0 ano de escolaridade 46 (31.3\%) alunos, o $8.040(27.2 \%)$ e o $9.061(41.5 \%)$. Os alunos distribuem-se entre os 12 e os 19 anos $(M=14.7 ; D P=1.58)$, existindo quatro com 18 anos e dois com 19. No 7.0 ano de escolaridade apresentam uma média etária de 13,5 anos ( $D P=1.01)$, no $8.914,6(D P=1.38)$ e no 9. o uma média de 15.8 ( $D P=1.35)$.

\subsection{Instrumentos}

Para avaliação dos processos autorregulatórios da aprendizagem foi aplicado o Inventário de Processos de Autorregulação da Aprendizagem (IPAA; ROSÁRIO et al, 2010), baseado no modelo de Zimmerman (2000). Este é constituído por nove itens representativos das três fases do processo de autorregulação da aprendizagem: planificação (e.g., Faço um plano antes de começar a fazer um trabalho. Penso no que vou fazer e no que é preciso para o completar), execução (e.g., Durante as aulas ou no meu estudo em casa, penso em coisas concretas do meu comportamento para mudar e atingir os meus objetivos) e avaliação (e.g., Comparo as notas que tiro com os meus objetivos para aquela disciplina). Os itens são apresentados num formato tipo Likert de 5 alternativas, desde 1 (nunca) até 5 (sempre). Os valores de consistência interna variam entre .77 e .85 . No presente estudo obtiveram-se valores similares que variaram entre .74 e .82 .

Para avaliar a disrupção percebida pelos alunos no seu ambiente de aprendizagem, foi aplicado o Questionário de Comportamentos Disruptivos Percebidos (CAPELA, 2003) constituído por 13 itens representativos de três fatores ou dimensões, a Distracção (e.g., distraem os colegas com palermices, por exemplo, quando saem do lugar para afiar o lápis ou para deitar um papel no lixo perturbam os colegas que estão no caminho), a Agressão (e.g., insultam com palavrões os colegas e as famílias) e a Transgressão de Regras (e.g., usam os telemóveis para enviar mensagens durante as aulas). No que se refere à classificação das respostas, usou-se, igualmente, uma escala de formato Likert de 5 pontos, desde 1 (nunca) até 5 (sempre). Os valores de consistência interna variam entre .59 e .75 e na presente investigação obtiveram-se valores superiores, que variaram entre .84 e .89 .

\subsection{Procedimentos}

Após a autorização da direção da escola, foram aplicados os questionários aos alunos em J unho de 2010, tendo sido assegurada a confidencialidade das respostas. O tratamento estatístico dos dados 
foi realizado com base no programa PASW Statistics 18/AMOS18 (ARBUCKLE, 2009; LOWE; WINZAR; WARD, 2007).

\subsection{Técnicas de análise de dados}

Relativamente à amostra, houve a preocupação de considerar cinco a dez respondentes por parâmetro no modelo (HAIR; ANDERSON; TATHAM; BLACK, 1998). Optou-se, também, por manter os outliers, uma vez que a estatística descritiva (desvio-padrão, curtose e assimetria) se mostrou, ainda, adequada.

Recorrendo a modelos de equações estruturais (ULLMAN; BENTLER, 2004), pesquisámos a validade preditiva, analisando as relações dos construtos avaliados com variáveis que os investigadores têm frequentemente referido como teoricamente associadas. Esta técnica de modelação de equações estruturais possibilita avaliar as relações causais entre variáveis inferidas (não diretamente observadas) através de um conjunto de variáveis observadas que servem de marcadores de cada variável inferida ou latente (BYRNE, 1994).

O modelo causal descrito é ao mesmo tempo projecto, construção e medição (MAlA, 1996). Por esta razão, podem ser consideradas quatro fases na sua estruturação. Em primeiro lugar, a fase de especificação do modelo que consiste na construção, pelo investigador, do modelo causal que estrutura o fenómeno em análise, escolhendo as variáveis mais importantes que melhor o explicam. Este modelo está baseado numa teoria que o antecede. Na presente investigação, quer os modelos de medida, quer os modelos de equações estruturais, foram delineados tendo como alicerce 0 racional teórico inerente às investigações dos construtos em estudo (BANDEIRA; ROCHA; SOUZA; DEL PRETTE; DEL PRETTE, 2006; HABER; GLATZER, 2009; MORÁN, 2004; VEIGA, 2008). Este modelo está representado pictograficamente por um diagrama causal (cf. figura 1), especificando as variáveis e a direção das influências causais.

Em segundo lugar, o objetivo é a identificação do modelo, que consta da análise das condições para que ocorra uma só solução para os parâmetros. Visto que estamos na presença de um sistema de equações que contém incógnitas, temos de certificar a possibilidade de obter uma solução, única ou não, para estas incógnitas (BOLLEN, 1989; MACCALLUM, 1995).

Em terceiro lugar, a finalidade consiste em estimar os parâmetros estruturais das equações, ou seja, dos coeficientes que representam as relações entre as variáveis. Neste estudo, o processo de estimação será concretizado em consonância com o método de máxima verosimilhança $(M L)$, que é o método mais recomendado na literatura (BRECKLER, 1990; MAIA, 1996, NúÑEZ, 1992). O cálculo dos 
parâmetros estimados foi efectuado com o suporte do programa informático atrás referido, que nos apresenta os modelos usados a partir de matrizes de efeitos e de covariância. Por fim, é referida a avaliação do modelo que é executada através dos indicadores estatísticos que possibilitam concluir a adequabilidade do enunciado estabelecido anteriormente.

$\mathrm{Na}$ avaliação dos modelos causais tentámos determinar se o modelo hipotetizado explica as relações existentes na matriz dos dados empíricos. A avaliação dos modelos será concretizada em duas etapas: a primeira relativa ao ajustamento global e a segunda ao ajustamento local. A análise do ajustamento global do modelo seguirá duas fases: a análise do ajustamento do modelo e a leitura dos dados relativos à comparação do modelo (MACCALLUM; WEGENER; UCHINO; FABRIGAR, 1993).

O ajustamento global do modelo representa o grau em que o modelo estabelecido se ajusta à matriz de covariância dos dados. A apreciação do modelo será feita com base nos dados estatísticos e índices mais habitualmente utilizados, nomeadamente: $x^{2} / g l$; GFI; AGFI; CFI; TLI e RMSEA (BYRNE, 2001; SCHREIBER; NORA; STAGE; BARLOW; KING, 2006).

Utiliza-se, frequentemente, o valor obtido a partir da divisão do $x^{2}$ do modelo pelo número de graus de liberdade $\left(x^{2} / g l\right)$, considerando um ajustamento aceitável do modelo quando este valor é inferior a 5 (GILES, 2002), embora este critério não seja pacífico entre os investigadores. Marsh e Hocevar (1985) sugeriram que um valor entre 2 e 5 indicaria um ajustamento aceitável, enquanto Byrne (1989) afirmou que valores superiores a 2 não deveriam ser aceites.

O índice GFI (Goodness-of-Fit Index), introduzido por Jöreskog e Sörbom (1983), compara a matriz de covariância da amostra utilizada com uma matriz de covariância estimada para a população, isto é, que parâmetros poderíamos esperar de replicações do estudo em diferentes amostras. O seu valor está compreendido entre 0 e 1 , sendo a proximidade de 1 uma medida de um bom ajuste. O AGFI (Adjusted Goodness-of-Fit Index) expressa, tal como o anterior, a quantidade de variância e covariância explicada, ajustada aos graus de liberdade do modelo. Valores iguais ou superiores a .90 nestes índices são normalmente considerados indicadores de ajustamento do modelo. Estes dois índices podem ser classificados como de ajustamento absoluto por não compararem o modelo hipotetizado com qualquer outro (HU; BENTLER, 1995).

O RMSEA é um índice que dá a ideia da diferença existente entre a matriz de variância e covariância da amostra e a matriz do modelo obtido, partindo do pressuposto que o modelo é adequado. Valores inferiores a .05 indicam um bom ajuste (BROWNE; CUDECK, 1993; HU; BENTLER, 1999). 
O TLI (Tucker-Lewis Index) possibilita comparar o modelo estimado com um modelo teórico nulo, ou seja, visa determinar se todos os indicadores são associados a um único fator latente (HAIR; ANDERSON; TATHAM; BLACK, 2005). Valores iguais ou superiores a .95 indicam um ajuste robusto.

O CFI (Comparative Fit Índex) coloca o modelo estimado algures num continuum de valores que oscilam entre 0 e 1 , indicando o zero um mau ajuste e o 1 o ajuste perfeito. Este índice, desenvolvido por Bentler (1990), compara o modelo estimado com um modelo completamente independente, no qual não existe nenhuma relação entre variáveis, com base no procedimento de máxima verosimilhança. É estimado a partir da diferença entre a unidade e o coeficiente entre a não-centralidade dos parâmetros calculados nos modelos a avaliar e de base. Este índice é importante pelo seu poder de ajuste em modelos estimados em amostras com um número reduzido de indivíduos. Valores iguais ou superiores a .95 são considerados indicadores de um bom ajuste (HARDY; BRYMAN, 2004; HU; BENTLER, 1990).

\section{Resultados}

Após a recolha dos dados, fez-se a sua aplicação no modelo (cf. Figura 1), tendo como referência as disciplinas de Língua Portuguesa e Matemática para definir o construto Notas. No modelo assumimos, como já foi referido, que a Disrupção percebida influencia a Autorregulação da aprendizagem e que esta tem impacto nas notas dos alunos. Foi assumido também que algumas variáveis exógenas (sexo, ano de escolaridade, número de reprovações, horas de estudo e metas escolares) também exercem influência na disrupção percebida dos alunos. As relações causais que foram hipotetizadas neste modelo derivam direta ou indiretamente da revisão de literatura já anteriormente explicitada. Para a medição dos construtos Disrupção percebida e Autorregulação da aprendizagem foram utilizados como indicadores os respectivos fatores, que nos permitiram obter um modelo com um ajuste robusto. 


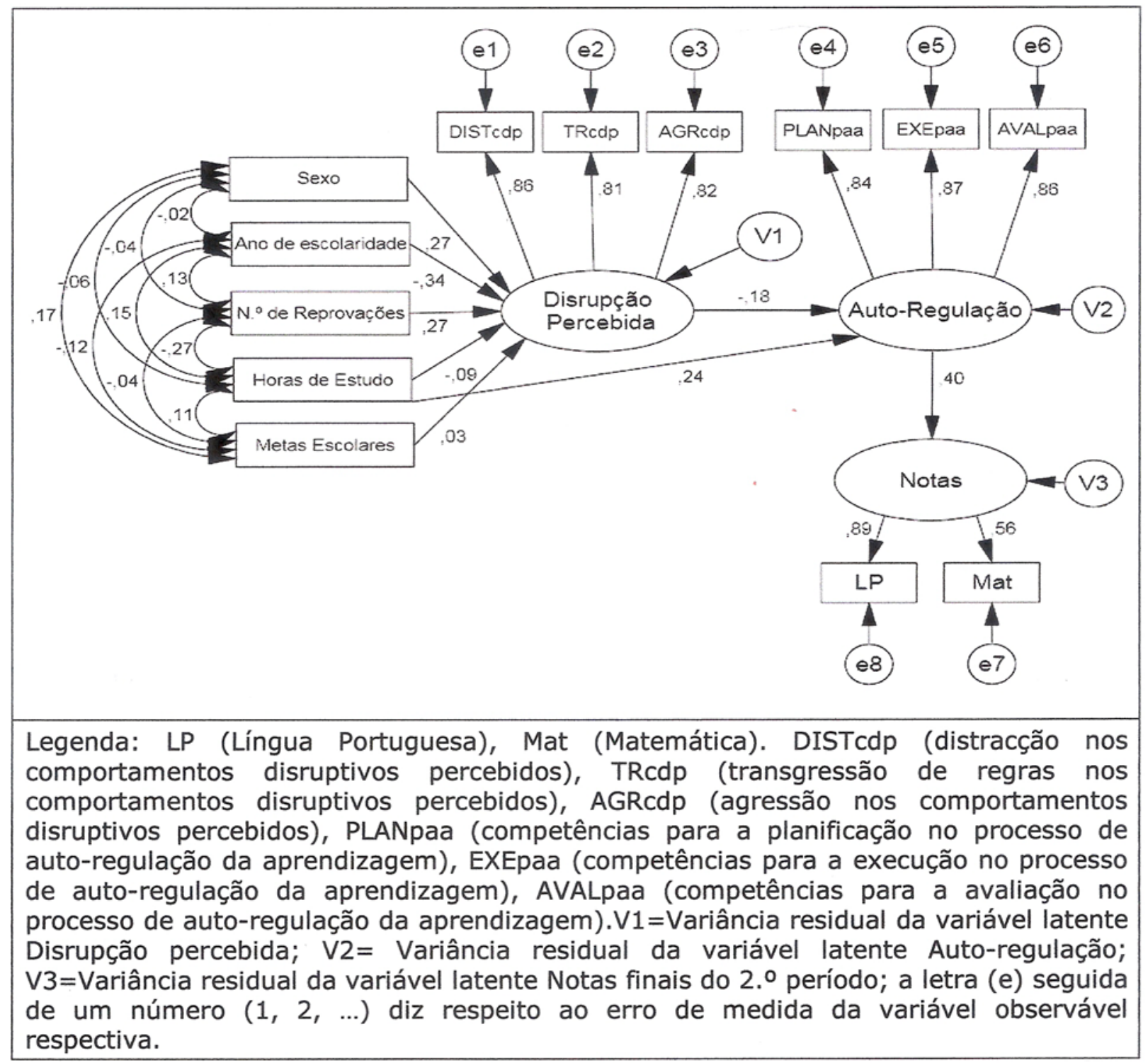

Figura 1. Especificação Pictórica do Modelo de Relações Causais com os Valores Estandardizados

De seguida serão indicados alguns aspectos essenciais da estatística descritiva. Na Tabela 1 , podem observar-se os dados descritivos (média, desvio-padrão, assimetria e curtose) correspondentes às variáveis incluídas no modelo de equações estruturais.

Um dos pressupostos importantes nos modelos de equações estruturais é que a distribuição de qualquer variável seja normal relativamente a qualquer valor das restantes variáveis, o qual implica que qualquer combinação linear das variáveis seja normal. Dado que o maximum likelihood pode produzir resultados distorcidos quando esta assunção é violada (WEST; FINCH; CURRAN, 1995), foi examinada a distribuição de cada uma das variáveis em relação à sua curtose e assimetria. Tomando como critério o estabelecido por 
Curran, West e Finch (1996), que entendem que apenas os valores de assimetria superiores a três e de curtoses superiores a dez não devem ser considerados, nenhuma das variáveis da amostra revela valores perto de tais critérios (cf. Tabela 1), pelo que parece justificado proceder à estimação do ajuste do modelo mediante ML.

\section{Tabela 1}

Estatística Descritiva (Média, Desvio-padrão, Assimetria e Curtose) Correspondente às Variáveis Incluídas no Modelo de Equações Estruturais

\begin{tabular}{lcccc}
\hline & M & DP & Assimetria & Curtose \\
\hline N.o de Reprovações & .57 & .81 & 1.485 & 2.148 \\
Horas de Estudo & 2.69 & 2.92 & 1.557 & 2.298 \\
Comportamentos Disruptivos Percebidos & & & & \\
$\quad$ Distracção & 13.73 & 5.45 & .335 & -.475 \\
$\quad$ Transgressão de regras & 10.70 & 3.90 & .444 & .024 \\
$\quad$ Agressão & 8.86 & 4.57 & .962 & .123 \\
Auto-regulação & & & & \\
$\quad$ Planificação da auto-regulação da aprendizagem & 10.65 & 2.77 & -.792 & .842 \\
$\quad$ Execução da auto-regulação da aprendizagem & 9.60 & 3.07 & -.231 & -.398 \\
$\quad$ Avaliação da auto-regulação da aprendizagem & 10.37 & 3.11 & -.564 & -.078 \\
Rendimento escolar & & & & \\
$\quad$ Língua Portuguesa & 2.67 & .72 & .477 & .563 \\
$\quad$ Matemática & 2.67 & .84 & .892 & .327 \\
\hline
\end{tabular}

Seguidamente analisaremos o modelo de equações estruturais em estudo. A Figura 1 especifica o modelo hipotetizado para os alunos da amostra. As relações causais possíveis são as constantes no modelo conforme as hipóteses delineadas na parte da Introdução.

A avaliação dos resultados da contrastação do modelo de equações estruturais, como já foi referido, é baseada em dois critérios: nível global de ajustamento do modelo e significância dos coeficientes de regressão calculados. Os índices de bondade de ajustamento global do modelo proposto poder-se-ão considerar robustos $\left[\mathrm{X}^{2}(\mathrm{gl})=53.009(52) ; \mathrm{p}=.435 ; \quad \mathrm{X}^{2} / \mathrm{gl}=1.019 ; \mathrm{GFI}=.951 ; \mathrm{AGFI}=.913 ;\right.$ $\mathrm{CFI}=.998 ; \mathrm{TLI}=.997 ; \mathrm{RMSEA}=.012$ ], confirmando a hipótese de que 0 modelo proposto representa as relações entre as variáveis existentes na nossa matriz empírica. 
Por outro lado, da análise da Tabela 2 e da Figura 1 pode concluir-se que as hipóteses que orientaram as especificações foram todas confirmadas, no entanto as hipóteses 4 e 5 não se apresentam estatisticamente significativas. Assim, verifica-se que: $\mathrm{H} 1$ - $\mathrm{O}$ sexo feminino revela uma melhor percepção dos comportamentos disruptivos relativamente ao sexo oposto $(a=.27 ; \mathrm{p}<.001) ; \mathrm{H} 2$ - Os alunos do 9.0 ano de escolaridade revelam menor percepção dos comportamentos disruptivos $(a=-.34 ; p<.001) ; \mathrm{H} 3$ - Os alunos com maior número de reprovações têm uma melhor percepção dos comportamentos disruptivos $(a=.27 ; \mathrm{p}<.01) ; \mathrm{H} 4$ - Um maior investimento no tempo de estudo, por parte dos alunos, influencia negativamente a percepção dos comportamentos disruptivos dos mesmos ( $a=-.09 ; p=n . s.) ; H 5$ - Os alunos que revelam metas escolares mais ambiciosas percepcionam melhor os comportamentos disruptivos ( $a=.03 ; p=n . s.) ; \mathrm{H} 6$ - Os alunos que apresentam uma melhor percepção sobre os comportamentos disruptivos são menos autorregulados na sua aprendizagem $(\beta=-.18 ; \mathrm{p}<.05) ; \mathrm{H} 7$ - Os alunos mais autorregulados na sua aprendizagem apresentam melhores notas escolares $(\beta=.40 ; p<.01) ; H I M ~-~ O s ~ a l u n o s ~ q u e$ investem mais horas no estudo apresentam uma maior frequência de comportamentos autorregulatórios na sua aprendizagem $(a=.24$; $\mathrm{p}<.01)$.

Da análise dos resultados das hipóteses formuladas, é de realçar que na sua maioria os valores de regressão de uma variável sobre a outra são significativos. Refira-se, ainda, que as variáveis Metas escolares e Horas de estudo exercem pouca influência na Disrupção percebida dos alunos, devido aos baixos valores de regressão.

Quanto às variáveis exógenas, da análise da Tabela 2, poder-se-á ainda mencionar que o sexo feminino reprova menos ( $a=-.04$; $\mathrm{p}=$ n.s.), apresenta metas escolares mais ambiciosas ( $\mathrm{a}=.17$; $p<.05)$, mas estudam menos $(a=-.06 ; p=n . s$.$) . Contudo, os alunos$ do 9.0 ano reprovam mais $(a=.13 ; p=n . s$.$) , apresentam metas$ escolares menos ambiciosas ( $a=-.12 ; p=n . s$.$) , no entanto estudam$ mais do que os seus colegas dos $7 .^{\circ}$ e $8 .^{\circ}$ anos de escolaridade (a $=.15 ; p=n$.s.). Nesta sequência os alunos que mais reprovam são os que investem menos tempo no seu estudo pessoal $(a=-.27 ; p<.01)$, apresentando também metas escolares menos ambiciosas ( $\mathrm{a}=-.04$; $\mathrm{p}=$ n.s.). Por sua vez os alunos que investem mais tempo no seu estudo têm igualmente metas escolares mais ambiciosas $(a=.11$; p=n.s.). De salientar que todas estas relações não são estatisticamente significativas, à excepção do número de reprovações e as horas de estudo e entre o sexo e as metas escolares. 


\section{Tabela 2}

Resultados da Contrastação da Estrutura de Covariância (Valor e Erro Estimado e Nível de Significância) Hipotetizado para a Amostra

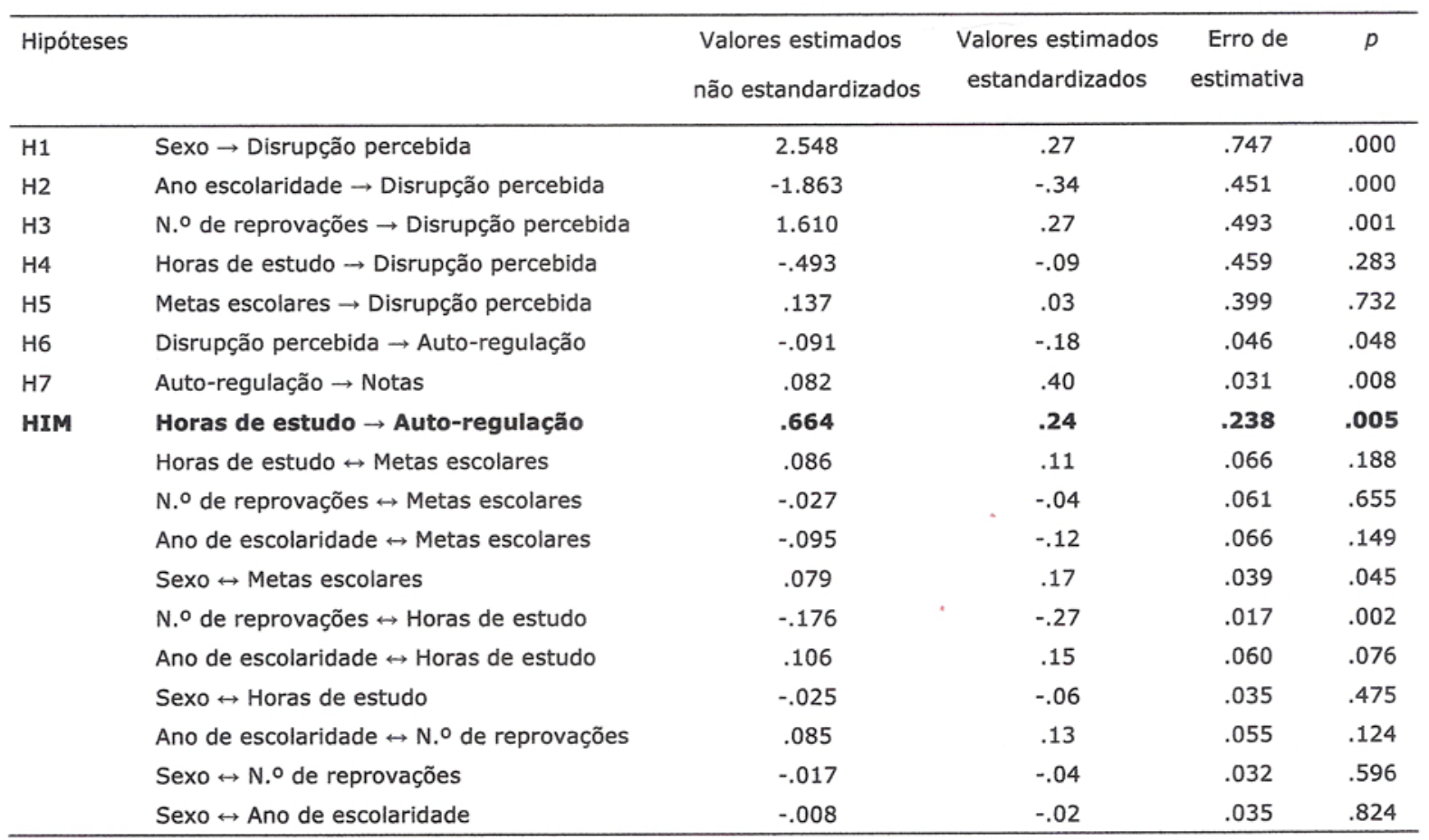

Relativamente às correlações múltiplas quadradas, estas indicam que as variáveis mais explicadas no modelo são a Disrupção percebida $(27.3 \%)$ e as Notas (16.3\%) e a menos explicada pelas variáveis em presença, embora relevante, é a Autorregulação da aprendizagem $(11.1 \%)$.

\section{Discussão e conclusões}

Uma das características importantes a assinalar no modelo desenvolvido por Zimmerman e colaboradores, centra-se no fato de tanto permitir explicar as realizações e adaptações de sucesso, como as disfunções (problemas afetivos, motivacionais e cognitivos) que poderão eventualmente surgir nos processos autorregulatórios. Esta característica reveste-se, assim, de um enorme significado para os educadores e professores, porque fornece um leque variado de informações que pode ser utilizado no contexto de sala de aula, optimizando o ensino/aprendizagem. Como referem Oliveira e Marinho- 
Araújo (2009), intervir nas concepções das ações e práticas dos professores e educadores é uma maneira de desenvolver mudanças nesses profissionais e nos próprios alunos, ajudando, assim, a rever os seus conceitos e práticas, reajustar suas prioridades, intenções e objetivo e reconsiderar o seu papel na formação dos alunos. Neste sentido, a prática docente deverá fornecer a estrutura e o apoio para encorajar o comportamento auto-regulado.

Os dados obtidos sugerem que os alunos com processos autorregulatórios mais agilizados percepcionam níveis de disrupção inferiores na escola $(p<.05)$. Provavelmente não se sentem incomodados com 0 tipo de comportamentos disruptivos, pois, habitualmente, os comportamentos agressivos acontecem na escola em sítios e ocasiões a que estes alunos facilmente têm acesso e frequentam propositadamente.

A literatura realça a relação entre a adequação comportamental dos alunos e as vivências do seu percurso escolar, demonstrando uma diferenciação da disrupção, à medida que se avança nos anos de escolaridade (CAPELA, 2003; ESTRELA, 1986), o que vem de encontro aos dados obtidos na presente investigação $(p<.001)$.

Porém, há várias respostas quanto à problemática das variações dos comportamentos disruptivos ao longo dos anos de escolaridade, quer devido às eventuais alterações de vida dos alunos, quer porque são escassos os estudos longitudinais apropriados para esclarecer a natureza da sua progressão. Era objetivo deste estudo, também, conhecer a relação entre o número de reprovações e as percepções de disrupção. Da análise dos dados podemos verificar que o número de reprovações apresenta um impacto significativo na percepção da disrupção dos alunos $(p<.01)$. Pelo contrário não encontramos uma associação estatisticamente significativa entre as horas de estudo e as metas escolares com a disrupção percebida.

Relativamente ao rendimento escolar dos alunos este está dependente de uma autorregulação eficaz da aprendizagem, como é referido em alguns estudos referenciados na literatura (ROSÁRIO; MOURÃO; SOARES; CHALETA; GRÁCIO; NÚÑEZ; GONZÁLEZ-PIENDA, 2005; ZIMMERMAN, 2002), o que mais uma vez é confirmado pelos nossos resultados $(p<.01)$. Esta regulação ativa implica o controle e escolha dos recursos ao seu alcance, para que o aluno vivencie ambientes facilitadores da aprendizagem, de acordo com os contextos sociais (HACKER; DUNLOSKY; GRAESSER, 2009). Assim, os alunos autorreguladores apresentam-se como agentes ativos $\mathrm{e}$ 
estruturadores do conhecimento, regulando a sua cognição, motivação e comportamento para atingir objetivos e melhorar realizações. Percepcionam, assim, uma maior competência que os ajuda a garantir os seus níveis de motivação e posterior utilização de estratégias autorregulatórias. Pelo contrário, os alunos menos autorregulados tendem a evitar as oportunidades de aprendizagem, evidenciam uma autoeficácia percebida mais baixa, bem como se envolvem menos nas atividades de estudo (ZIMMERMAN; KITSANTAS, 1997). Seria, pois, de esperar uma relação entre os padrões de autorregulação dos alunos e as percepções de disrupção, tal como se verifica nos resultados encontrados.

No que diz respeito à relação entre o número de reprovações e os comportamentos disruptivos percepcionados a literatura existente relaciona significativamente insucesso escolar e disrupção. Fonseca, Taborda e Formosinho (2000), num estudo longitudinal, referem que os alunos retidos apresentam índices mais elevados de comportamento anti-social do que os colegas sem reprovações. Na realidade, estes autores sublinham que o comportamento escolar inadequado está muito associado à distração, ao desinteresse e a uma determinada transgressão do comportamento esperado do aluno. Pelo fato, têm uma percepção mais apurada dos comportamentos disruptivos, o que vem de encontro aos resultados obtidos neste estudo e parecem coerentes com a literatura existente.

Nesta sequência, o insucesso escolar apresenta uma relação inversamente proporcional com a competência de autorregulação da aprendizagem (LOURENÇO, 2008; ROSÁRIO et al, 2005; ZIMMERMAN, 2002) e é considerada pelos autores um fator de risco para a indisciplina na aula. Note-se, no entanto, que alguns investigadores (ESTRELA, 1986; VEIGA, 1995) são de opinião que os alunos com melhores classificações aceitam melhor a autoridade, são mais atentos, originam menos conflitos, são mais responsáveis, colaboram mais com os colegas, são mais cuidadosos com o material escolar, respeitam mais os professores e seguem mais as suas indicações, podendo, por alheamento, ou defensivamente, não registar este comportamento. Contrariamente, um percurso escolar marcado pelo insucesso poderá influenciar fortemente a personalidade dos alunos no seu autoconceito e nas aspirações acadêmicas, mesmo que demonstrem indiferença (LOOS, 2003).

No que diz respeito à Disrupção percebida, esta está explicada, no modelo especificado, diretamente pelas variáveis exógenas sexo, ano de escolaridade, número de reprovações, horas de estudo e metas escolares em $27.3 \%$. Por seu lado, a variável Notas está explicada 
diretamente pela Autorregulação em 16.3\%, o que nos levará a pensar que os alunos mais autorregulados obtêm mais sucesso escolar. No que diz respeito à variável Autorregulação esta é explicada diretamente pelas variáveis Disrupção percebida e Horas de estudo em $11.1 \%$. As três variáveis endógenas que integram 0 modelo (variáveis dependentes mediadoras) não se encontram igualmente explicadas pelos efeitos estimados no modelo. $O$ conjunto de relações causais especificadas apresenta um nível explicativo pouco robusto relativamente a alguns construtos em estudo. Estes resultados, não comprometendo a validade do modelo que ajustou de uma forma muito satisfatória, representam, no entanto, uma importante limitação quanto à capacidade explicativa dos efeitos entre as variáveis. Contudo, as possíveis variações nas mesmas parecem estar determinadas também por outras variáveis não incluídas no modelo. Estes dados, incontornáveis, sugerem a necessidade de reespecificarmos o modelo, procurando outras variáveis que, influindo significativamente nas variáveis dependentes, aumentem a variância explicada das variáveis endógenas. Dessa forma, ganharíamos na compreensão dos comportamentos disruptivos dos alunos da amostra.

Assim, não se pretende responder totalmente ao problema em questão, quer pelos limites de algumas variáveis em estudo, quer pela necessidade de replicar esta investigação, tendo em consideração outras amostras sociodemográficas com características diferenciadas, contemplando alunos quer do ensino público quer do ensino privado, para que a generalização dos resultados possa ser mais efectiva.

Finalmente, o que se procurou foi construir um modelo onde fosse possível reflectir sobre a relação existente entre a disrupção percebida, a autorregulação da aprendizagem, o rendimento académico e algumas variáveis sociodemográficas, partindo, essencialmente, das vivências quotidianas do aluno no espaço escolar e da sua dinâmica atitudinal perante o sucesso académico. Este estudo constitui um primeiro subsídio na abordagem da disrupção percebida pelos alunos e suas relações com a autorregulação da aprendizagem e sucesso escolar; esperamos, em trabalhos futuros, poder alargar o contributo neste domínio académico.

\section{Referências}

ARBUCKLE, J. L. Amos 18.0 User's Guide. Crawfordville: Amos Development Corporation, 2009.

BANDEIRA, M.; ROCHA, S. S.; SOUZA, T. M. P.; DEL PRETTE, Z. A. P.; DEL PRETTE, A. Comportamentos problemáticos em estudantes do 
ensino fundamental: características da ocorrência e relação com habilidades sociais e dificuldades de aprendizagem. Estudos de Psicologia, Natal, v. 11, n. 2, p. 199-208, 2006.

BANDURA, A. Social Cognitive Theory in Cultural Context. Applied Psychology: An International Review, Hoboken, v. 51, n. 2, p. 269-290, 2002.

BENTLER, P. M. Comparative fit indexes in structural models. Psychological Bulletin, Berkeley, v. 107, p. 238-246, 1990.

BISQUERRA, R. Métodos de investigación educativa. Guia prático. Barcelona: CEAC, 1989.

BOEKAERTS, M.; CORNO, L. Self-Regulation in Classroom: A Perspective on Assessment and Intervention. Applied Psychology: An I nternational Review, Hoboken, v. 54, n. 2, p. 199-231, 2005. BOLLEN, K. A. Structural equations with latent variables. New York: Wiley, 1989.

BRECKLER, S. J. Applications of covariance structure modelling. In Psychology: Cause for concern? Psychological Bulletin, Berkeley, v. 52, p. 260-271, 1990.

BRODY, G. H.; STONERNAN, Z.; FLOR, D. Parental religiousity, farnily processes and youth cornpetence in rural, two-parent African Arnerican farnilies. Developmental Psychology, Michigan, v. 32, p. 696-706, 1996.

BROWNE, M. W.; CUDECK, R. Alternative ways of assessing model fit. In BOLLEN, K. A.; LONG, J. S. (Eds.). Testing Structural Equation Models. Newbury Park, CA: Sage, 1993. p. 445-455.

BYRNE, B. M. A primer of LISREL: Basic Applications and Programming for Confirmatory Factor Analytic Models. New York: Springer-Verlag, 1989.

BYRNE, B. M. Structural equation modelling with EQS and EQS/ Windows: Basic concepts, applications and programming. Thousand Oaks, California: Sage Publications, 1994.

BYRNE, B. M. Structural Equation Modeling With AMOS - Basic Concepts, Applications, and Programming. New Jersey: Lawrence Erlbaum, 2001.

CAPELA, Z. A disrupção em sala de aula e as competências auto-regulatórias da aprendizagem. 2003. 208f Tese (Mestrado em Educação) - Instituto de Educação e Psicologia da Universidade do Minho, Braga.

CURRAN, P. J.; WEST, S. G.; FINCH, J. F. The robustness of test statistics to nonnormality and specification error in confirmatory factor analysis. Psychological Methods, San Diego, v. 1, p. 16-29, 1996.

EPSTEIN, M. H.; KINDER, D.; BURSUCK, B. The academic status of adolescents with behavioural disorders. Behavioural Disorders, London, v. 14, n. 3, p. 157-165, 1989. 
ESTRELA, M. T. Une étude sur l'indiscipline en classe. Lisboa: INIC, 1986.

FONSECA, A. C.; TABORDA, S.; FORMOSINHO, M. Retenção escolar precoce e comportamentos anti-sociais. Revista Portuguesa de Pedagogia, Coimbra, v. 24, n. 1-3, p. 323-340, 2000.

GILES, D. C. Advanced research methods in psychology. New York: Routledge, 2002.

HABER, J.; GLATZER, J. Bullying - manual anti-agressão. Alfragide: Casa das Letras, 2009.

HACKER, D. J.; DUNLOSKY J.; GRAESSER, A. C. Handbook of Metacognition in Education. Mahwah, New Jersey: Erlbaum, 2009. HAIR, J. F.; ANDERSON, R. E.; TATHAM, R. L.; BLACK, W. C. Multivariate Data Analysis with Readings, 5th Edition. Englewood Cliffs, NJ: Prentice Hall, 1998.

HAIR, J.; ANDERSON, R.; TATHAM, R.; BLACK, W. Análise multivariada de dados. Porto Alegre: Bookman, 2005.

HARDY, M.; BRYMAN, A. Handbook of data analysis. Thousand Oaks, CA: Sage, 2004.

HU, L.-T.; BENTLER, P. M. Evaluating model fit. In: Hoyle, R. H. (Ed.). Structural equation modelling: concepts, issues, and applications. Thousand Oaks, CA: Sage, 1995, p. 76-99.

HU, L.-T.; BENTLER, P. M. Cut off criteria for fit indexes in covariance structure analysis: conventional criteria versus new alternatives.

Structural Equation Modeling: A Multidisciplinary Journal, Thousand Oaks, CA, v. 6, p. 1-55, 1999.

JÖRESKOG, K. G.; SÖRBOM, D. LISREL - 6 User's Reference Guide. Mooresville, IN: Scientific Software, 1983.

LOOS, H. Atitude e desempenho em matemática, crenças autoreferenciadas e família: uma path analysis. 2003. $305 \mathrm{f}$ Tese (Doutoramento em Psicologia da Educação) - Faculdade de Educação da Universidade Estadual de Campinas, São Paulo.

LOURENÇO, A. A. Processos Auto-Regulatórios em Alunos do 3.o Ciclo do Ensino Básico: Contributo da Auto-Eficácia e da Instrumentalidade. 2008. 228f Tese (Doutoramento em Educação) Instituto de Educação e Psicologia da Universidade do Minho, Braga. LOWE, B.; WINZAR, H.; WARD, S. Essentials of SPSS for Windows versions 14 \& 15: a business approach. South Melbourne, Victória: Thomson Learning Australia, 2007.

MACCALLUM, R. C. Model specification: Procedures, strategies, and related issues. In: HOYLE, R. H. (Ed.). Structural equation modelling: Concepts, issues, and applications. Thousand Oaks, CA: Sage, 1995, p. 16-36.

MACCALLUM, R. C.; WEGENER, D. T.; UCHINO, B. N.; FABRIGAR, L. The problem of equivalent models in applications of covariance structure analysis. Psychological Bulletin, Berkeley, v. 114, n. 1, p. 185-199, 1993. 
MAIA, J. A. R. Um discurso metodológico em torno da validade de construto: posição de um lisrelita. In: IV CONFERÊNCIA INTERNACIONAL SOBRE AVALIAÇÃO PSICOLÓGICA: FORMAS E CONTEXTOS, Actas, Braga: APPORT, 1996, p. 43-50.

MARSH, H. W.; HOCEVAR, D. The application of confirmatory factor analysis to the study of self-concept: first and higher order factor structures and their invariance across age groups. Psychological Bulletin, Berkeley, v. 97, p. 562-582, 1985.

MCCOMBS, B. L. Self-regulated learning and academic achievement. In: ZIMMERMAN, B. J.; SCHUNK, D. H. (Eds.). Self-regulated learning and academic achievement: Theory, research and practic. New York: Springer-Verlag, 1989, p. 51-82.

MERRETT, F. E.; WHELDALL, K. Classroom behavior problems which junior school teachers find most troublesome. Educational Studies, San Diego, v. 10, p. 87-92, 1984.

MORÁN, H. Autoconcepto, enfoques de aprendizaje y rendimiento académico en alunnos de formación profesional de Galicia. 2004. 257f Tese (Doutoramento em Psicologia) Universidade da Coruña, A Coruña.

NÚÑEZ, J. C. EI autoconcepto: Características estructurales, diferencias evolutivas inter e intra-individuales y su relación com el rendimiento académico en alumnos de 6 a 11 años. 1992. 395f Tese (Doutoramento em Psicologia) - Facultad de Psicologia, Universidad de Oviedo, Oviedo.

OLIVEIRA, C. B. E.; MARINHO-ARAÚJ O, C. M. Psicologia escolar: cenários atuais. Estudos e Pesquisas em Psicologia, Rio de J aneiro, v. 9, n. 3, p. 648-663, 2009.

PAIVA, M. O. A.; LOURENÇO, A. A. Comportamentos Disruptivos versus Rendimento Académico: uma abordagem com modelos de equações estruturais. Psicologia, Educação e Cultura, Pedroso, v.13, n.2, p. 283-306, 2009.

ROSÁRIO, P. Estudar o estudar: (Des)venturas do Testas. Porto: Porto Editora, 2004.

ROSÁRIO, P.; GONZÁLEZ-PIENDA, J. A.; CEREZO, R.; PINTO, R.; FERREIRA, P.; LOURENÇO, A.; PAIVA, O. Eficacia del programa «(Des)venturas de Testas» para la promoción de un enfoque profundo de estudio. Psicothema, Oviedo, v. 22, n. 4, p. 828-834, 2010.

ROSÁRIO, P.; LOURENÇO, A. A.; PAIVA, M. O.; NÚÑEZ, J. C.; GONZÁLEZ-PIENDA, J.; VALLE, A. Inventário de processos de autoregulação da aprendizagem (IPAA). In: MACHADO, C.; GONÇALVES, M. M.; ALMEIDA, L. S.; SIMÕES, M. R. (Coords.). Instrumentos e Contextos da Avaliação Psicológica. Coimbra: Almedina, 2010, p. 159-174.

ROSÁRIO, P.; MOURÃO, R.; SOARES, S.; CHALETA, E.; GRÁCIO, L.; NÚÑEZ, J. C.; GONZÁLEZ-PIENDA, J. Trabalho de casa, tarefas 
escolares, auto-regulação e envolvimento parental. Psicologia em Estudo, Maringá, v. 10, n. 3, p. 343-351, 2005.

ROSÁRIO, P.; NÚÑEZ, J.; GONZÁLEZ-PIENDA, J. Stories that show how to study and how to learn: an experience in Portuguese school system. Electronic Journal of Research in Educational Psychology, Almería, v. 1, p. 131-144, 2004.

ROSÁRIO, P.; SOARES, S.; MOURÃO, R.; NÚÑEZ, J. C.; GONZÁLEZPIENDA, J., SIMÕES, F.; PINA, F. Disrupção percebida e Autoregulação da aprendizagem no Ensino Básico. Psicologia e Educação, Pedroso, v. 4, n. 1, p. 77-90, 2005.

SCHREIBER, J. B.; NORA, A.; STAGE, F. K; BARLOW, E. A.; KING, J. Reporting Structural Equation Modeling and Confirmatory Factor Analysis Results: A Review. The J ournal of Educational Research, Bahawalpur, v. 99, n. 6, p. 323-337, 2006.

SCHUNK, D. H. Self-regulation of self-efficacy and attributions in academic settings. In: SCHUNK, D. H.; ZIMMERMAN, B. J. (Eds.). Self-regulation of learning and performance: Issues and educational applications. Hillsdale: Erlbaum, 1994, p. 75-99.

SCHUNK, D. H.; ERTMER, P. A. Self-regulation and academic learning, self-eficacy enhancing interventions. In: BOEKAERTS, M.; PINTRICH. P.; . ZEIDNER, M. (Eds.). Handbook of self-regulation. San Diego, NY: Academic Press, 2000, p. 631-649.

ULLMAN, J. B.; BENTLER, P. M. Structural Equation Modeling. In: HARDY, M.; BRYMAN, A. (Eds.). Handbook of data analysis. London: Sage, 2004, p. 431-458.

VEIGA, F. H. Escala de disrupção escolar inferida pelos professores (EDEI): construção e validação. Revista Portuguesa de Pedagogia, Coimbra, v. 29, n. 1, p. 99-118, 1995.

VEIGA, F. H. Indisciplina e violência na escola: Práticas comunicacionais para professores e pais. 2a ed. Coimbra: Almedina, 2001.

VEIGA, F. H. Disruptive Behavior Scale Professed by Students (DBSPS): Development and Validation. International Journal of Psychology and Psychological Therapy, Almería, v. 8, n. 2, p. 203-216, 2008.

WEST, S. G.; FINCH, J. F.; CURRAN, P. J. Structural equation models with non-normal variables: Problems and remedies. In: HOYLE, R. (Ed.). Structural Equation Modeling: Concepts, Issues and Applications. Newbury Park, CA: Sage, 1995, p. 55-75.

WINNE, P. H.; NESBIT, J. C. The Psychology of Academic Achievement. Annual Review of Psychology, Palo Alto, v. 61, p. 653-678, 2010.

ZIMMERMAN, B. J. Attaining self-regulation. A social cognitive perspective. In: BOEKAERTS, M.; PINTRICH, P.; ZEIDNER, M. (Eds.). Hanbook of self-regulation. New York, San Diego: Academic Press, 2000, p. 13-39. 
ZIMMERMAN, B. J. Achieving self-regulation. The trial and triumph of adolescence. In: PAJARES, F.; URDAN, T. (Eds.). Academic motivation of adolescents. Greewhich, CT: Information Age Publishing, 2002, p. 1-27.

ZIMMERMAN, B. J.; KITSANTAS, A. Development phases in selfregulation: Shifting from process to outcome goals. Journal of Educational Psychology, Memphis, v. 89, p. 29-36, 1997.

ZIMMERMAN, B. J.; MARTINEZ-PONS, M. Development of a Structured Interview for Assessing Student Use of Self-Regulated Learning Strategies. American Educational Research Journal, Winter, Washington, v. 23, n. 4, p. 614-628, 1986.

ZIMMERMAN, B. J.; MARTINEZ-PONS, M. Construct validation of a strategy model of student self-regulated learning. Journal of Educational Psychology, Memphis, v. 80, p. 284-290, 1988.

ZIMMERMAN, B. J.; MARTINEZ-PONS, M. Student differences in selfregulated learning: Relating grade, sex, and giftedness to selfefficacy and strategy use. Journal of Educational Psychology, Memphis, v. 82, n. 1, p. 51-59, 1990.

\section{Endereço para correspondência}

Maria Olímpia Almeida de Paiva

Avenida Camilo 4300-096 Porto, Portugal

Endereço eletrônico: investigar2010@gmail.com

Abílio Afonso Lourenço

Avenida Camilo 4300-096 Porto, Portugal

Endereço eletrônico: investigar2010@gmail.com

Recebido em: 10/12/2010

Reformulado em: 09/02/2011

Aceito para publicação em: 14/02/2011

Acompanhamento do processo editorial: Adriana Benevides Soares

Notas

* Professora Titular.

**Professor Titular. 